Preprints of the

Max Planck Institute for

Research on Collective Goods

Bonn 2010/28

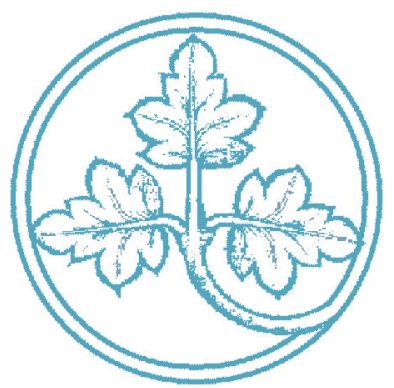

On the Prevalence of Framing Effects Across

Subject-Pools in a Two-

Person Cooperation Game

Sebastian J. Goerg

Gari Walkowitz

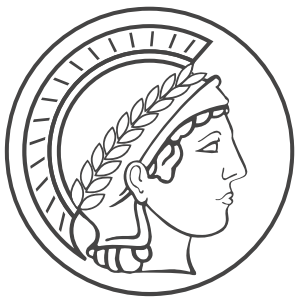




\section{On the Prevalence of Framing Effects Across Subject-Pools in a Two-Person Cooperation Game}

Sebastian J. Goerg / Gari Walkowitz

June 2010 


\title{
On the Prevalence of Framing Effects Across Subject-Pools in a Two-Person Cooperation Game **
}

\author{
Sebastian J. Goerg ${ }^{\mathrm{a}, \mathrm{c}}$ and Gari Walkowitz ${ }^{\mathrm{b}, \mathrm{c}, *}$
}

July 5, 2010

\begin{abstract}
In this experimental study, involving subjects from Abu-Dis (West Bank), Chengdu (China), Helsinki (Finland), and Jerusalem (Israel), we test for a presentation bias in a two-person cooperation game. In the positive frame of the game, a transfer creates a positive externality for the opposite player, and in the negative frame, a negative one. Subjects in Abu-Dis and Chengdu show a substantially higher cooperation level in the positive externality treatment. In Helsinki and Jerusalem, no framing effect is observed. These findings are also reflected in associated first-order beliefs. We argue that comparisons across subject-pools might lead to only partially meaningful and opposed conclusions if only one treatment condition is evaluated. We therefore suggest a complementary application and consideration of different presentations of identical decision problems within (cross-cultural) research on subject-pool differences.
\end{abstract}

Keywords: framing of decision problems, methodology, subject-pool differences

JEL Classification:A13, C72, C91, F51, Z13

\footnotetext{
${ }^{* *}$ We are grateful to Mai Abdeen, Zeina Barakat, Mohammed Dajani, Hong Geng, Heike Hennig-Schmidt, Shani Kuna, Jan Meise, Markku Verkasalo, and Chaoliang Yang for supporting the conduct of the experiments in Abu-Dis, Chengdu, Helsinki, and Jerusalem. We also wish to thank Andrea Ahlgrimm, Jeffrey Carpenter, Brian Cooper, Jieyao Ding, Marion Eberlein, Christoph Engel, Simon Gächter, Johannes Kaiser, Jan-Erik Lönnqvist, Reinhard Selten, Philipp Wichardt, Eyal Winter, and the participants of the ESA meetings in Hong Kong, Atlanta, and Shanghai, the IZA and Cologne-Bonn workshops on behavioral economics, both held in Bonn in 2006, and the Sino-German Summer School 2007 in Bonn for valuable comments and discussions. Financial support by the Deutsche Forschungsgemeinschaft is gratefully acknowledged.

${ }^{a}$ Max-Planck-Insititute for Research on Collective Goods, Bonn

${ }^{b}$ Department of Management, University of Cologne

${ }^{c}$ Laboratory for Experimental Economics (BonnEconLab), University of Bonn

*Corresponding author: gari.walkowitz@uni-koeln.de

Herbert-Lewin-Str. 2, 50931 Cologne, Germany, Tel.: +49 2214701842; fax: +49 2214701849.
} 


\section{Introduction}

A vast body of literature demonstrates that different presentations of the same decision task can induce a so-called framing effect: behavior changes, although the underlying information and decisions remain essentially the same. Early investigations of pure framing effects have shown that variations in the form of presentation - of cooperation problems as a whole or of specific variables - manipulate level and mode of cooperation (Pruitt, 1967; Selten and Berg, 1970). Differently labeled decision tasks (e.g., game title, players' actions, player types, etc.) can also lead to divergent and non-consistent behavior (c.f., Tversky and Kahneman, 1981; Elliott, Hayward, and Canon, 1998; Burnham, McCabe, and Smith, 2000; Liberman, Samuels, and Ross, 2004). Moreover, further contributions have revealed that subjects' behavior can be influenced by the presentation of the same essential information as positive or negative. This type of framing is commonly known as valence framing. In this broad domain, studies dealing with public goods games creating either positive externalities (public good) or negative externalities (public bad) are well established (c.f. Fleishman, 1988; Andreoni, 1995; Sonnemans, Schram, and Offerman, 1998; Willinger and Ziegelmeyer, 1999; Cookson, 2000; and Park, 2000; Dufwenberg, Gächter, and Hennig-Schmidt, 2008). ${ }^{2}$ Results from these publications in general suggest that experimental designs enabling positive externalities are aligned with significantly higher cooperation levels compared to setups allowing for negative externalities. $^{3}$

This paper intends to analyze subject-pool affiliation as one factor leading to different und conflicting levels of cooperation dependent on game presentation forms with either positive or negative externalities. As shown by several authors choosing subjects from different countries promises substantial cross-societal variation (see, e.g., Roth, Prasnikar, Okuno-Fujiwara, and Zamir, 1991; Oosterbeek, Sloof, and van de Kuilen (2004), Herrmann, Thöni, and Gächter, 2008). To maximize chances of observing behavioral differences across subject-pools, we conducted an experimental study in different subject pools - Abu-Dis (West Bank), Chengdu (China), Helsinki (Finland), and Jerusalem (Israel) - holding the type of subject pool (uni-

\footnotetext{
${ }^{2}$ See also Levin, Schneider, and Gaeth (1998) and Abbink and Hennig-Schmidt (2006) for comprehensive reviews on framing literature and framing types.

${ }^{3}$ Brewer and Kramer (1986), McCusker and Carnevale (1995), and Sell, Chen, Hunter-Holmes, and Johansson (2002) found an effect that went into the opposite direction. In a British subject-pool Cubitt, Drouvelis, and Gächter (2008) do not find a significant difference in contributions across game frames; neither do Dufwenberg et al. (2008) in their neutral label game.
} 
versity students) constant. The societies of our subject-pools differ according to widely used criteria developed by social scientists and, partly, in geographical distance. ${ }^{4}$ In addition to these classifications, the historical and political background of Israelis and Palestinians ${ }^{5}$ - who live next to each other - makes them a promising testbed for investigating the link between subject-pool affiliation and cooperative behavior.

Since cooperation in situations with positive or negative externality is crucial for human interaction from an individual perspective as well as from a societal point of view, we will also separately compare behavior under both conditions across subject-pools to evaluate the validity of findings. We will further show that our experimental approach and the awareness of the impact of subject-pool differences on frame perception have important implications. Formally identical bargaining setups might be perceived differently, evoke deviant beliefs in different subject-pools, and lead to divergent behavior.

Cross-societal studies conducted so far typically apply experimental designs with one form of presentation. Possible, unintentionally induced presentation effects - although not the focus - are not considered (e.g., Anderson, Rodgers and Rodriguez, 2000; Henrich, Boyd, Bowles, Camerer, Fehr, Gintis, and McElreath, 2001; Buchan, Croson, and Johnson, 2004). ${ }^{6}$ To the best of our knowledge there exist only two studies taking a cross-societal perspective of framing effects into account. The first work, a questionnaire study by Levin, Gaeth, Evangelista, Albaum, and Schreiber (2001), involves Americans and Australians. Therein, American subjects claimed to reduce a significantly higher amount of red meat consumption if the negative consequences of not reducing (i.e., a higher risk of developing cancer) were stressed compared to a treatment in which the positive consequences of reducing were emphasized. On the contrary, Australian subjects did not respond differently to the two frames. In a second study,

\footnotetext{
${ }^{4}$ As Inglehart, Basañez, and Moreno (2001) point out, different behavioral standards and norms are particularly likely if countries vary in their religious heritage. Our subject pools are located in countries that bear different religious heritages: Islam in Palestine; Buddhism, Taoism, and Confucianism (although not a religion in a narrower sense) in China; Christianity in Finnland; and Judaism in Israel. Moreover, contrary to geographically distant Chinese and Palestinians who are collectivistic and high-context societies (i.e., they use high-context messages in routine communication, in which words and word choices are very important. Few words can communicate a complex message very effectively to an in-group), Finns and Israelis - who are also located far from each other - live in more individualistic and low-context societies (i.e, the communicator needs to be more explicit and the value of a single word is less important) (Hall, 1976; Hofstede, 2001).

${ }^{5}$ At the moment, a Palestinian state does not exist. Most of our subjects are formally citizens of the states of Israel and Jordan. Nevertheless, we will refer to them as Palestinians to ease the notation.

${ }^{6}$ See, for a specific international overview of public goods and commons dilemma studies, Cardenas and Carpenter (2004).
} 
Sell et al. (2002) investigated the consistency and direction of framing effects across different countries. They found very similar patterns of cooperation both in the United States and in China. In both subject-pools, group members were more cooperative when facing resource goods dilemmas compared to a situation where they were confronted with a standard public goods game. We will extend the approach of Levin et al. (2001) and Sell et al. (2002) with regard to a more extensive cross-subject-pool analysis and a discussion of the behavioral and - more importantly - methodological consequences of our findings. ${ }^{7}$

For our study, we conducted two series of experiments applying two frames of a simple two-person continuous prisoner's dilemma game which represent different presentations of the same - strategically equivalent - decision task. That is, in both cases individuals must choose between a maximization of their own profit or to cooperate at some personal cost to increase the joint payoff. Individuals can give up an immediate benefit to sustain a resource for the other player's use. Thus, in one treatment, action creates a positive externality for the matched player. On the contrary, in our second treatment, action results in a negative externality. Like a public goods dilemma, our first treatment is a problem of contribution. Only with positive contributions is an increase in efficiency achieved. Similarly, our second treatment, like a commons dilemma, is a problem of consumption. The lower the share of personal consumption, the higher is the efficiency. Any consequentialist theory, such as expected utility theory, suggests that these two types of presentations are equivalent because the underlying game-structures are strategically equivalent and have the same payoff consequences. However, giving and taking are psychologically different actions and findings from one set of studies may not be generalized to the other set; generally between different subject-pools and especially in a cross-societal environment (Brewer and Kramer, 1986; Fleishman, 1988). This fact makes our experimental framework an appropriate tool to study presentation effects across subject-pools.

Our West Bank and Chinese data show that game presentation can significantly influence decision makers' actions and associated first-order beliefs. In the positive externality con-

\footnotetext{
${ }^{7}$ Since we have not conducted more than one experiment per society we implicitly rely on the assumption that there are no differences within societies and that the only difference is between subject-pools. Yet, some experimental evidence supports this assumption: Gächter and Herrmann (2009) and Herrmann and Thöni (2009) conducted experimental studies in more than one subject pool in Switzerland and Russia and found no differences within societies but only between them. Future research should address this methodological point on a broader international scale.
} 
dition, substantially more cooperation is manifested in both subject-pools compared to the situation with negative externality. In contrast, the experiments conducted in Helsinki and Jerusalem yielded different results. There, on an aggregate level, no significant presentation effect could be detected. In all subject-pools, neither the Nash equilibrium nor the social optimum is reached.

Comparing the level of cooperation under each of our two conditions across subjectpools yields opposite conclusions about cooperative behavior in the different subject-pools. While subjects in Abu-Dis and Chengdu are more cooperative in the treatment with positive externality, behavior in the treatment with negative externality is more cooperative in Helsinki and Jerusalem.

Our results shed new light on the impact of presentation conditioned by preferences and social norms in different subject-pools embedded in different societies. Framing effects are not robust. Therefore, we will argue that for deriving a conclusion about a subject-pool's (cooperative) behavior, different presentations of logically identical experimental setups should be considered and evaluated adequately. We believe that this is of particular importance when discussing cross-cultural evidence.

\section{Experimental framework: A two-person cooperation game}

The two applied game frames both represent a straightforward two-person continuous prisoner's dilemma game in which subjects can choose an individual level of cooperation from a given range of possible actions. ${ }^{8}$ Thus, in contrast to the classical prisoner's dilemma game, the question whether to cooperate or to defect is not a binary choice. In the first game-frame (PDP), a player's decision creates a positive externality to the matched player's payoff, while in the second game-frame (PDN), it induces a negative externality. In the next subsection, we will describe both the PDP-frame and the PDN-frame in detail.

\subsection{Game-frame with positive externality (PDP)}

At the beginning, two randomly matched players $i$ and $j$ obtain an integer initial endowment $X=X_{i}=X_{j}$. Each player then has the opportunity to transfer an integer part $a$ of $X$,

\footnotetext{
${ }^{8}$ Please refer to Appendix A for further details on the prisoner's dilemma game and public goods game nature of the game.
} 
nothing, or the entire amount $X$ to the opposite player. Both players choose $a \in\{0, \ldots, X\}$ simultaneously. Each amount $a$, which is transferred to the paired player, will be multiplied by factor $k>1$ yielding an efficiency gain by transferring a positive amount $a$. Players' payoffs consist of the initial endowment $X$ minus the transferred amount $a$ plus the obtained and $k$-multiplied amount $a$ transferred by the opposite player. Formally, player $i$ 's payoff function is given by:

$$
\pi_{i}^{P D P}=X_{i}-a_{i}^{P D P}+k \cdot a_{j}^{P D P}, \text { with } a_{i}^{P D P}, a_{j}^{P D P} \in\{0,1, \ldots, X\}, \text { and } k>1
$$

The payoff of the opposite player $j$ is calculated analogously. The only Nash equilibrium is $a_{i}^{*}=a_{j}^{*}=0$. Player $i$ anticipates player $j$ 's choice $a_{j}^{P D P}=0$ and will therefore also choose $a_{i}^{P D P}=0$. The collective optimal choice is $\hat{a}_{i}=\hat{a}_{j}=X$ since it maximizes the joint payoff $\Pi^{P D P}=\pi_{i}+\pi_{j}$.

\subsection{Game-frame with negative externality (PDN)}

The design of the PDN-frame is equivalent to the first version of the game, but instead of choosing an amount $a$ which is transferred to the opposite player, decision makers must choose an integer which is transferred from the other player. Again two players $i$ and $j$ interact simultaneously. Initially, both receive an endowment $X=X_{i}=X_{j}$. Each player then has the opportunity to transfer an integer part $a$, nothing, or the entire amount $X$ from the matched player. Thus, again, both players simultaneously choose $a \in\{0, \ldots, X\}$. The difference $X-a$, which is respectively not transferred, will be multiplied with $k>1$. Hence, by transferring low amounts or nothing, efficiency increases. In contrast to the PDP-frame, the amount $a$, which is transferred, is not multiplied. Players' payoffs are determined by the multiplied difference of their initial endowments $X$ and the amount $a$ taken by the opposite player, and the amount $a$ which players take away from the counterpart. Formally, player $i^{\prime} s$ payoff function is given by:

$$
\pi_{i}^{P D N}=\left(X_{i}-a_{j}^{P D N}\right) \cdot k+a_{i}^{P D N}, \text { with } a_{i}^{P D N}, a_{j}^{P D N} \in\{0,1, \ldots, X\}, \text { and } k>1
$$

Player $j$ 's payoff is calculated analogously. The only Nash equilibrium is $a_{i}^{*}=X_{j}$ and $a_{j}^{*}=X_{i}$. Player $i$ anticipates player $j$ 's choice $a_{j}^{P D N}=X_{i}$ and will therefore also choose $a_{i}^{P D N}=X_{j}$. The optimal collective choice is $\hat{a}_{i}=\hat{a}_{j}=0$ since it maximizes the joint payoff $\Pi^{P D N}=\pi_{i}+\pi_{j}$. 


\subsection{Equivalence of the two game-frames}

In both versions of the game, player $i$ 's payoff $\pi_{i}$ consists of two parts - a self-determined component $\pi_{i A}$ and a part $\pi_{i B}$ resulting from player $j$ 's actions. Therefore, the total payoff of player $i$ can be stated as: $\pi_{i}=\pi_{i A}+\pi_{i B}$. Player $i$ 's self-determined payoff fraction in the PDP-frame is the amount $X_{i}^{P D P}-a_{i}^{P D P}$ which is not given to the other player. In the PDN-frame, it is the amount $a_{i}^{P D N}$ that is taken away from the other player.

The amount $a_{j}^{P D P} \cdot k$ is the amount which player $i$ receives from the other player in the PDP-frame. In the PDN-frame, the amount determined by the other player is the payoff fraction $\left(X_{i}^{P D N}-a_{j}^{P D N}\right) \cdot k$ that the other player leaves to player $i$.

This illustrates that there are one-to-one mappings $f_{i}$ and $f_{j}$ of player $i$ 's or $j$ 's strategies $a_{i}$ and $a_{j}$, respectively, in the PDP-frame onto the strategy spaces for $i$ and $j$ in the PDNframe such that $a_{i}, a_{j}$ and $\left(f_{i}\left(a_{i}\right), f_{j}\left(a_{j}\right)\right)$ yield the same payoffs in the PDP-frame and the PDN-frame, respectively.

\section{Experimental procedures}

The experiments were conducted between May 2006 and February 2007. The sessions in Abu-Dis were run at the Al-Quds University located in the West Bank, close to the city of Jerusalem. We collected the Chinese data at the Sichuan University in Chengdu. Finnish data were gathered at the University of Helsinki and observations for Jerusalem were obtained at the RatioLab of the Hebrew University in Jerusalem. In all universities students from different departments participated. ${ }^{9}$

In Abu-Dis and Jerusalem, each student received a fixed payment of 25 NIS upon showing up for the experiment. In Chengdu [Helsinki], each subject was paid 20 YUAN [4 EURO]. At each university both versions of the game were played as one-shot games, applying the paper and pencil method. We have chosen one-shot games to avoid confounding framing effects with strategical issues and to elicit behavior that is only conditioned to subjects' experiences

\footnotetext{
${ }^{9}$ In Chengdu, Helsinki, and Jerusalem, only subjects with very limited experimental experience were recruited (excluding previous collaborations in trust game, prisoner's dilemma, gift exchange, or public goods game experiments) for participation. Palestinian subjects had no experimental experience. The median age in Abu-Dis [Chengdu, Helsinki, Jerusalem] was 22 [23,24,25], and 55\% [48\%,69\%,40\%] females participated, respectively. We checked for possible effects of age and gender. We could not find any significant influences, neither for each subject-pool separately nor for the complete sample of observations.
} 
Table 1

Overview over Treatments, Locations and Observations

\begin{tabular}{llll}
\hline Session & Treatment & Location & \# subjects \\
\hline & & & \\
1 & PDP & Abu-Dis & 20 \\
2 & PDN & Abu-Dis & 20 \\
3 & PDP & Jerusalem & 20 \\
4 & PDN & Jerusalem & 20 \\
5 & PDP & Chengdu & 20 \\
6 & PDN & Chengdu & 20 \\
7 & PDP & Helsinki & 21 \\
8 & PDN & Helsinki & 21 \\
& & & 142 \\
\hline
\end{tabular}

and perceptions within their specific reference groups. Table 1 displays sessions, treatment conditions, and locations.

Experiments were run by local helpers who were comprehensively instructed and supported by the authors, who in turn, were present but stayed in the background. The motivation for this procedure was to minimise potential experimenter demand effects which arguably would be much higher if one of the authors would have run the experiment because of the cultural attribution - foreigners might be perceived differently across locations - that subjects might have made in this case. In addition, if a local runs the experiments there are no language issues and culture is not primed. ${ }^{10}$

Instructions were written in neutral language, strictly avoiding terms like 'give', 'take', or 'cooperation'. In fact, we neither labeled nor put disposable strategies into positive or negative light which should enhance the validity of our findings. They differed between treatments only by the direction of the conducted transfer. Accordingly, transfers were to be realized either to player $j$ or from player $j$. This procedure ensured that only the technical presentation and not the wording or further frames could influence subjects' behavior. Dependent on the location, the instructions were either given in Arabic, Chinese, Finnish, or Hebrew. ${ }^{11}$

Subjects were endowed with $X=10$ Talers in the opening of every game. ${ }^{12}$ The multiplier

\footnotetext{
${ }^{10}$ This procedure was also implemented by Herrmann et al. (2008).

${ }^{11}$ To avoid translation errors regarding the task and the procedure, instructions were translated by native speakers from German into the corresponding language and afterwards back-translated into German by a different person (Brislin, 1970). For instructions, see Appendix B.

${ }^{12}$ Taler $=$ experimental currency. During the experiment all transfers were made in Taler. The exchange rate from Taler to NIS [YUAN, EURO] was 1 Taler $=2.5$ NIS [1.5 YUAN, 0.5 EURO]. We adjusted expected
} 
$k$ was fixed with $k=2$. The individual payoff in the Nash equilibrium was 10 Talers for each player. The Pareto optimum outcome generated 20 Talers, respectively. In the run of the experiment, participants received no feedback on matched player's decisions.

After running the experiment, two questionnaires were handed out. In the first questionnaire, we asked participants for their first-order beliefs on the behavior of the matched player. ${ }^{13}$ Correct beliefs were rewarded with an additional Taler. The second questionnaire covered socio-demographic questions. At the end of the session, the outcome for each participant was calculated, converted into the local currency, and paid out.

\section{Results}

The basis of our analysis is the level of cooperation exhibited by the participants in both treatment conditions. In the PDP-frame, it is the transferred amount to the other player $\left(a^{P D P}\right)$, and in the PDN-frame, it is the amount left to the other player $\left(10-a^{P D N}\right)$. We will first compare treatment-dependent cooperation levels and associated first-order beliefs across subject-pools and check for consistency among treatments. In a second step, we will first investigate potential framing effects within our subject-pools and then contrast actual framing effect magnitudes across subject-pools.

\subsection{Treatment-dependent cooperation across subject-pools}

In PDP, the highest average cooperation level is observed in Abu-Dis (7.10), followed by Chengdu (5.50), Jerusalem (4.40), and Helsinki (3.67). Nearly the same sequence holds for first-order beliefs on cooperation: highest contributions are expected in Abu-Dis (6.05), followed by Chengdu (4.55), Helsinki (3.57), and Jerusalem (3.40). A Kruskal-Wallis test shows that cooperation levels and associated first-order beliefs differ significantly across subjectpools in $\operatorname{PDP}(p=.004, p=.027$; both two-sided $) .{ }^{14}$ Hence, when we exclusively look at the positive externality frame, which is commonly done in subject-pool comparisons, we find

\footnotetext{
hourly payoffs to the average wage per hour of a local student helper.

${ }^{13}$ We are aware of the fact that stated beliefs can be biased by prior decisions that have already been undertaken. However, since actual unbiased decisions are more valuable for our analysis, we applied this procedure.

${ }^{14}$ Pair-wise analyses reveal that contributions in Abu-Dis are economically and statistically higher than in Jerusalem and Helsinki $(p=.000, p=.005)$. Moreover, subjects in Chengdu are more cooperative than in Helsinki $(p=.084)$ (all Mann-Whitney-U test, two-sided).
} 
clear varieties (see Table 2 and Figure 1). This leads us to our first result:

Result 1: In the positive externalities treatment (PDP), cooperation levels differ significantly across subject-pools. The same holds for associated first-order beliefs.

Next, we investigate average cooperation levels in PDN across subject-pools. In PDN, the highest cooperation is found in Helsinki (4.67), followed by Jerusalem (4.55), and Chengdu (2.65) and Abu-Dis (2.65). We receive almost the same order when analyzing associated first-order beliefs: highest contributions are expected in Helsinki (3.67), followed by Jerusalem (3.40), Chengdu (3.20), and Abu-Dis (2.75) (see Table 2 and Figure 1). A Kruskal-Wallis test reveals that cooperation levels significantly differ across subject-pools in $\mathrm{PDN}(p=.053$, twosided). ${ }^{15}$ However, this does not hold for associated first-order beliefs $(p=.900$, two-sided $) .{ }^{16}$ Hence, we conclude:

Result 2: In the negative externalities treatment (PDN), cooperation levels significantly differ across subject-pools. Associated first-order beliefs are not statistically different across subject-pools.

\subsection{The impact of framing in different subject-pools}

We now explore whether there are differences in the impact of different game frames on cooperation levels and associated first-order beliefs across subject-pools to explain our previous findings.

In Abu-Dis [Chengdu], on average 7.10 [5.50] Talers were transferred to the opposite player in PDP. In the contrary, in PDN, only 2.65 [2.65] Talers were left to the opponent. The observed mean differences between the two treatments are highly significant in both locations ( $p=.000[p=.009]$, both Mann-Whitney-U test, two-sided). A look at the medians yields a similar result (see Figure 1). When looking at first-order beliefs we find a comparable pattern: in both locations, subjects on average expected higher cooperation in PDP (6.05 [4.55]) compared to PDN (2.75 [3.20]). In Abu-Dis, the difference in beliefs is highly significant $(p=.000)$ whereas in Chengdu, average beliefs do not significantly differ ( $p=.321$; all MannWhitney-U test, two-sided).

\footnotetext{
${ }^{15}$ Pairwise analyses show that cooperation in Helsinki is significantly higher than in Chengdu and Abu-Dis ( $p=.059$ and $p=.032$ ). The same holds for Jerusalem; here cooperation is also significantly higher compared to Chengdu and Abu-Dis ( $p=.045$ and $p=.077$ ) (all Mann-Whitney-U test, two-sided).

${ }^{16} \mathrm{In}$ all subject pools actions and associated beliefs are positively correlated, refer to Table 2 .
} 
Table 2

Average cooperation levels, associated first-order beliefs and correlation between beliefs and cooperation level per location

\begin{tabular}{|c|c|c|c|}
\hline Abu-Dis & Chengdu & Helsinki & Tomplom \\
\hline
\end{tabular}

Cooperation levels

$\begin{array}{lllll}\text { PDP } & 7.10(2.36) & 5.50(3.69) & 3.67(2.63) & 4.40(2.95) \\ \text { PDN } & 2.65(2.08) & 2.65(2.76) & 4.67(3.26) & 4.55(3.38) \\ \text { MWU } & p<.01 & p<.01 & p=.41 & p=.95\end{array}$

First-order beliefs

$\begin{array}{lllll}\text { PDP } & 6.05(2.89) & 4.55(3.90) & 3.57(2.79) & 3.40(2.50) \\ \text { PDN } & 2.75(2.34) & 3.20(3.21) & 3.67(3.38) & 3.40(3.14) \\ \text { MWU } & p<.01 & p=.32 & p=.88 & p=.97\end{array}$

Correlation First-order belief/Cooperation level

$\begin{array}{lllll}\text { Spearman } & \rho=.64 & \rho=.68 & \rho=.58 & \rho=.41 \\ \text { Significance } & p<.01 & p<.01 & p<.01 & p<.01\end{array}$

Numbers in paratheses denote standard deviations.

MWU: two-sided Mann-Whitney-U-test for the comparison between PDP and PDN.

Figure 1

Median levels of cooperation and associated first-order beliefs per treatment and subject-pool. Diamonds give means and corresponding error bars.

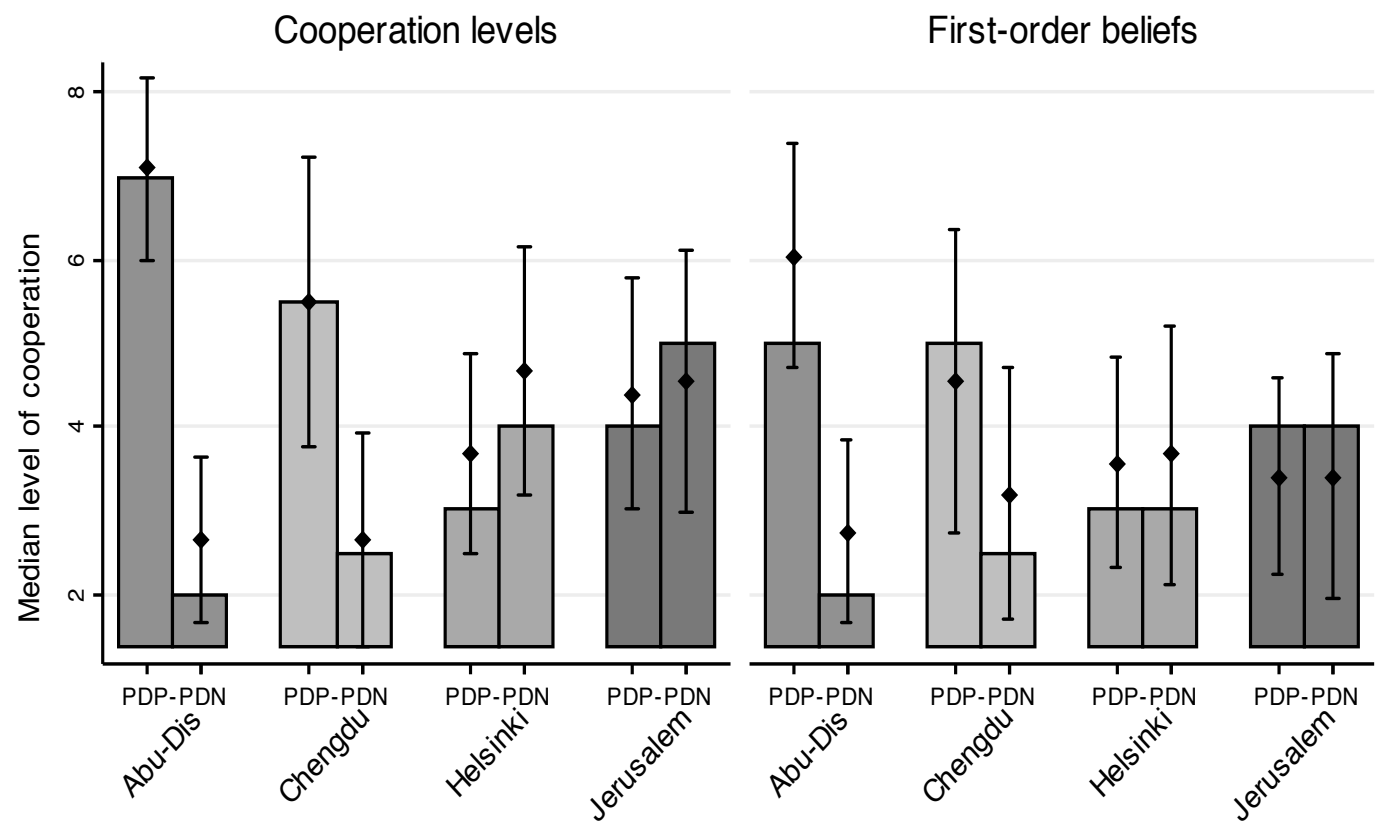


In Helsinki and Jerusalem, we observe a different picture. In Helsinki [Jerusalem], the shown average level of cooperation is slightly higher under PDN than in PDP. In PDP on average 3.67 [4.40] Talers were transferred to the other player compared to 4.67 [4.55] which were left in PDN. However, these differences are not statistically significant $(p=.407[p=$ .952]. The same finding holds for first-order beliefs: in Helsinki [Jerusalem], subjects on average expected similar cooperation in PDP (3.57 [3.40]), as compared to PDN (3.67 [3.40]). In both places, beliefs do not differ significantly $(p=.875[p=.973]$, all Mann-Whitney-U test, two-sided).

Our findings on the prevalence of framing effects illustrate that framing effects in cooperative behavior are not robust; in Abu-Dis and Chengdu, they are detectable, whereas in Helsinki and Jerusalem, no evidence for a presentation bias is found (cf. Table 2 and Figure 1). In addition, mean differences between PDP and PDN also differ across subject-pools. In order to quantify, these differences we now compare the exact magnitude of the detected framing effects in pairs between subject-pools - this is the difference in cooperation levels among PDP and PDN per location - by comparing the differences in differences between two locations and applying a statistical test to them. For this analysis we use a Monte-Carlo approximation of a two-sided permutation test with 100,000 draws. ${ }^{17}$ Our analysis shows that the effect magnitude in Abu-Dis [Chengdu] is significantly higher than in Helsinki $(p=.000$ $[p=.007])$ and in Jerusalem $(p=.000[p=.004])$. No differences in effect magnitudes can be found among Abu-Dis and Chengdu nor for a comparison between Helsinki and Jerusalem $(p=.222$ and $p=.539)$.

Taking our evidence on the impact of framing across subject-pools yields our third result:

Result 3: In Abu-Dis and Chengdu, subjects are significantly more sensitive to the gameframe than subjects from Helsinki and Jerusalem. In both subject-pools, cooperation is significantly higher under the PDP-condition than in the PDN-treatment. On the contrary, no evidence for a presentation effect in Helsinki and Jerusalem is observed.

Our results demonstrate that observed levels of cooperation found in one subject-pool

\footnotetext{
${ }^{17}$ We apply a test, which computes the probability for obtaining a sample with the same or a larger difference between the two effect magnitudes by randomly assigning each single action in PDP and PDN to one of the two locations, while at the same time keeping the condition constant. The resulting fraction of trials with larger or same differences functions as the $p$-value. This procedure is analogous to the one applied by Abbink and Rockenbach (2006). Cohen's $d$ could also be applied as a measure for the effect size per location. However, this yields only aggregate measures, which are not testable (Cohen's $d$ : Abu-Dis $d=1.99$; Chengdu $d=0.875$; Helsinki $d=0.337$; Jerusalem $d=0.047)$.
} 
broadly depend on the game presentation form. In addition and strikingly, the ordering of cooperation levels across subject-pools is almost reversed in the two treatment conditions: in PDP, we observe the highest level of cooperation in Abu-Dis and the lowest in Helsinki. On the contrary, in PDN we find most cooperation in Helsinki and least in Abu-Dis (together with Chengdu). This detected swapping of order, nicely captured by Figure 1, shows how sensitive cross-subject-pool comparisons can potentially be toward game frames - results derived from comparisons under one game frame do not necessarily hold for other frames of the same decision task. ${ }^{18}$ Consequently, we conclude the results section with our last result:

Result 4: The elicitation, occurrence, and comparison of cooperative behavior across different subject-pools substantially depends on the chosen game presentation. Therefore results may vary across game frames and subject-pools.

\section{Summary and Discussion}

The aim of this paper was to investigate the impact of framing on cooperative behavior dependent on subject-pool affiliation. Investigating two logically and strategically identical decision problems in an international setting we demonstrated that data obtained from only one presentation form might lead to only partly valid results and conclusions on subject-poolspecific behavior. This finding holds especially if results are compared and evaluated across subject-pools. Our results confirm and extend the work by Levin et al. (2001) and Sell et al. (2002).

Our results from Abu-Dis and Chengdu have shown that the formal presentation of a decision problem can influence subjects' actions substantially. The observed cooperation level is significantly higher when subjects create positive externalities toward each other compared to a situation in which resulting externalities are negative. In the positive condition, subjects from Abu-Dis and Chengdu are more willing to transfer higher amounts and thus voluntarily increase mutual welfare. On the contrary, in the negative condition, subjects leave relatively less to the counterpart.

Experiments run in Helsinki and Jerusalem yielded different results. There, aggregated

\footnotetext{
${ }^{18}$ One interesting detail, however, is, that average observed cooperation levels (i.e., the average cooperation levels over both presentations) do not differ significantly between locations. Average cooperation levels [firstorder beliefs] over both presentation forms are: Abu-Dis 4.88 [4.40], Chengdu 4.08 [3.88], Helsinki 4.17 [3,62] and Jerusalem 4.48 [3.40].
} 
subjects' actions appear to be externally unaffected across treatments in terms of the measured average outcome. No significant presentation effect could be verified. Finns and Israelis seem to show a similar behavioral pattern in both treatments. This finding is consistent with findings from Cubitt et al. (2008) and Dufwenberg et al. (2008) who conducted experiments with German and British subjects, respectively.

Our findings in Abu-Dis and Chengdu are in line with prior work on presentation effects in public goods games (e.g., Andreoni, 1995; Sonnemans et al., 1998; Willinger and Ziegelmeyer, 1999; and Park, 2000) and with studies on goal framing (e.g., Meyerowitz and Chaiken, 1987; Levin, Schneider and Gaeth, 1998). In these experiments, the negative formulation of an identical problem leeds to less cooperative behavior compared to a positive one.

The observed behavior could be connected to the concept of loss aversion and the so-called endowment effect, as introduced by Tversky and Kahneman (1981). To explain evident higher cooperation in take-dilemmas McCusker and Carnevale (1995) argue that give dilemmas involve decisions about current losses and take dilemmas about gains. Since people are motivated to avoid a loss more than they are motivated to obtain an equivalent gain they contribute more in the take game. Contrary to this link it is possible that our Palestinian and Chinese subjects perceive an amount taken away from them as a substantial loss, while they perceive an amount voluntarily given away as less of a loss or no loss. In other words, they are more sensitive to a loss induced by a second person, compared to a loss induced by themselves. As a consequence of this cognition, they might react much more sensitively to the threat of a possible loss induced by the right of the second player to take away any amount, as compared to the situation where they can determine themselves which amount to give away. To avoid this expected loss induced by the matched player, players take more from the matched player and, thus, cooperation is on a lower level in the PDN-frame as compared to the PDP-frame. This might deliver an explanation why, in this study, Palestinians and Chinese seem to obtain a higher benefit from doing a good deed rather than from not doing a bad deed. ${ }^{19}$

An alternative explanation for the observed behavior in Abu-Dis and Chengdu refers to the action itself. In the PDP-frame, action leads to cooperation, whereas under the PDN-

\footnotetext{
${ }^{19}$ Andreoni (1995) argues that utility of people increases if they perceive the act of transferring as doing something good ("warm-glow") and decreases when they perceive it as doing something bad ("cold-prickle").
} 
condition, the opposite holds. There, action results in competitive and less efficient behavior. The difference in the sensitivity toward the given frame might stem from a different attitude toward action depending on power to control, i.e. to decide how much to transfer. Galinsky, Gruenfeld, and Magee (2003) have shown that priming high power leads to increased action in a social dilemma regardless of whether that action had pro-social or anti-social consequences. Being primed with power incites participants both to give more to and take more from a commonly shared resource. The different perception of own power of Palestinians and Chinese - performing notably more action both in the PDP- and in the PDN-frame (14.45 [12.85] Talers are transferred in sum in both treatments) - and Finns and Israelis - showing a lower degree of action in both conditions (transferring 9.00 [9.85] Talers) - may deliver an approach to explain behavioral differences across subject-pools and potentially even cultures. Future research should address this issue by linking different concepts of self image (e.g., power perception, self esteem) and situational power to control to decisions.

Behavior in Jerusalem and Helsinki might be rooted in the fact that our subjects live in Western, more individualistic, and low-context societies. In such societies, ties between individuals are loose, people are expected to look out for themselves, and behavior and beliefs are spelled out explicitly with single words being perceived as less content-loaded (Hall, 1976; Hofstede, 2001). A series of studies has illustrated that individualists often behave more competitively and outcome-oriented in cooperation settings, compared to people from collectivistic and high-context subject-pools (e.g., Hemesath and Pomponio, 1998; Carpenter, Daniere, and Takahashi, 2004; Buchan, Johnson, and Croson, 2006; Chuah, Hoffmann, Jones, and Williams, 2007; Hennig-Schmidt, Li, and Yang, 2008). This finding is confirmed by our data if we only consider behavior under the positive externality condition which represents the commonly applied game frame in subject-pools located in different cultures. Further studies must address the cause for the similarity of behavior displayed under different presentation conditions: Do Israelis and Finns actually perceive the two games as presentation forms of the same decision problem, or do they apply different approaches leading to similar behavioral consequences and outcome?

One interesting question in this context is whether the framing of the game also affects beliefs and how beliefs may, in turn, condition subjects' outcomes as previously demonstrated by Dufwenberg et al. (2008), and Fischbacher and Gächter (2010). Hence, if different frames 
affect beliefs of different subject-pools differently and if beliefs guide behavior we might also find subject-pool differences in framing effects. Large subject-pool differences which generate well pronounced discrepancies in belief structures might therefore also enhance differences in actual behavior. As we have demonstrated, our findings on subjects' cooperative actions are largely reflected in subjects' first-order beliefs. In addition, as Table 2 in the results section indicates, actions and beliefs are significantly positively correlated. In fact, we can assume that in our cross-cultural setting behavior is to some extent guided by underlying first-order beliefs. Future studies should explicitly pick up the following issues: firstly, whether subjects' contributions are guided by existing belief structures in broader cross-cultural settings and, secondly, how differences in this conditional cooperation can be explained by variables typical to those societies included.

The findings of our study might confront cross-societal research with new challenges: comparing levels of cooperation under each of the conditions across subject-pools might lead to opposing conclusions about society-specific behavioral attitudes and their underlying motives. Our Palestinian and Chinese subjects display a relatively higher cooperation level and more positive beliefs on opponent players' contributions than involved Finns and Israelis when only the positive externalities condition is considered. On the contrary, Finnish and Israeli subjects cooperate relatively more and state substantially higher beliefs when only the negative externalities condition is regarded. These striking findings would not have been detected by the implementation of a mere positive framing of the cooperation task. Taking results from different presentations into account might not only enrich socio-economic theory, but also refine our experimental methodology. 


\section{References}

Abbink, K. and H. Hennig-Schmidt 2006: Neutral versus loaded instructions in a bribery experiment, Experimental Economics, Vol. 9, 103-121.

Abbink, K. and B. Rockenbach (2006): Option Pricing by Students and Professional Traders: A behavioral Investigation, Managerial and Decision Economics, 27 (6), 497-510.

Anderson, L. R., Y. V. Rodgers, and R. R. Rodriguez (2000): Cultural differences in attitudes toward bargaining, Economics Letters, Vol. 69, 45-54.

Andreoni, J. (1995): Warm-glow versus cold-prickle: The effects of positive and negative framing on cooperation in experiments, Quarterly Journal of Economics, 110(1), 1-21.

Bond, M.H., and K. Hwang (1986): The Psychology of the Chinese People, in M. H. Bond (Ed.): The social psychology of the Chinese people, Oxford University Press, Hong Kong, pp. 213-266.

Brewer, M.B., and R.M. Kramer (1986): Choice Behavior in Social Dilemmas: Effects of Social Identity, Group Size, and Decision Framing, Journal of Personality and Social Psychology, 50(3), 543-549.

Brislin, R. W. (1970): Back-translation for cross-cultural research, Journal of Cross-cultural Psychology, 1(3), 185-216.

Buchan, N., R. T. A. Croson, and E. J. Johnson (2004): When do fairness beliefs influence bargaining behavior: Experimental evidence from Japan and the United States, Journal of Consumer Research, Vol. 31, 181-190.

Buchan, N.R., E.J. Johnson, and R.T.A. Croson (2006): "Let's get personal: an international examination of the influence of communication, culture and social distance on other regarding preferences", Journal of Economic Behavior and Organization, 60(3), 373-398.

Burnham, T., K. McCabe, and V.L. Smith (2000): "Friend- or Foe: Intentionality Priming in an Extensive Form Trust Game, Journal of Economic Behavior and Organization, 43, 57-74.

Cardenas, J. C. and J. Carpenter (2004): An inter-cultural examination of cooperation in the commons, Middlebury College Working Paper, Middlebury College.

Carpenter J. P., A. Daniere, and L. Takahashi (2004): Cooperation, Trust and Social Capital in Southeast Asian Urban Slums, Journal of Economic Behavior and Organization, 55, 533-551.

Chuah, S.H., R. Hoffmann, M. Jones, and G. Williams (2007): Do Cultures Clash? Evidence from Cross-National Ultimatum Game Experiments, Journal of Economic Behavior and Organization, 64(1), 35-48.

Cohen, Y. and Y. Haberfeld (1998): Second-Generation Jewish Immigration in Israel: Have the Ethnic Gaps in Schooling and Earnings Declined? Ethnic and Racial Studies, Vol. 21, 507-528. 
Cookson, S. (2000): Framing effects in public good games, Experimental Economics, Vol. 3, 55-79.

Cubitt, R., M. Drouvelis, and S. Gächter (2008): Framing and Free Riding: Emotional Responses and Punishment in Social Dilemma Games, CeDEx Discussion Paper, No. 2008-02.

Dufwenberg, M., S. Gächter, H. Hennig-Schmidt (2008): The Framing of Games and the Psychology of Strategic Choice, BGSE Discussion Paper 15/2008, University of Bonn.

Elliott, C.S., D.M. Hayward, and S. Canon (1998): Institutional Framing: Some Experimental Evidence, Journal of Economic Behavior and Organization, 35, 455-64.

Fershtman, C. and U. Gneezy (2001): Discrimination in a segmented society: An experimental approach, The Quarterly Journal of Economics,116(1), 351-377.

Fischbacher and Gächter (2010): "Social Preferences, Beliefs, and the Dynamics of Free Riding in Public Good Experiments", American Economic Review, 100(1), 541-556.

Fleishman, J. A. (1988): The effects of decision framing and others' behavior on cooperation in a social dilemma, Journal of Conflict Resolution, 32(1), 162-180.

Gächter, S. and B. Herrmann (2009): Reciprocity, culture and human cooperation: previous insights and a new cross-cultural experiment, Philosophical Transactions of the Royal Society B, 364, March 2009, 791-806.

Gächter, S., H. Orzen, E. Renner, and C. Starmer (2009): Are Experimental Economists Prone to Framing Effects? A Natural Field Experiment. Journal of Economic Behavior and Organization, $70(3), 443-446$.

Galinsky, A.D., D.H. Gruenfeld, and J.C. Magee (2003): From Power to Action, Journal of Personality and Social Psychology, 85(3), 453-466.

Hall, E. T. (1976): Beyond Culture, New York: Doubleday.

Hemesath, M. and X. Pomponio (1998): Cooperation and Culture: Students From China and the United States in a Prisoner's Dilemma, Cross-Cultural Research, 32(2), 171-184.

Hennig-Schmidt, H., Z.-Y. Li, and C. Yang (2008): Why people reject advantageous offers - Nonmonotonic strategies in ultimatum bargaining: Evaluating a video experiment run in PR China, Journal of Economic Behavior and Organization, 65 (2), 373-384.

Henrich, J., R. Boyd, S. Bowles, C. Camerer, E. Fehr, H. Gintis, and R. McElreath (2001): In search of homo economicus: Behavioral experiments in 15 small- scale societies, American Economic Review, $91(2), 73-78$.

Herrmann, B. and C. Thöni (2009): Measuring conditional cooperation: a replication study in Russia, Experimental Economics, 12, 87-92. 
Herrmann, B., C. Thöni, and S. Gächter (2008): Antisocial Punishment Across Societies, Science, 319, 1362-1367.

Hofstede, G. (2001): Culture's Consequences: Comparing Values, Behaviors, Institutions and Organizations across Nations. Second Edition, Thousand Oaks, CA: Sage Publications.

Inglehart, R., M. Basañez, and A. Moreno (2001): "Human Values and Beliefs", University of Michigan Press, MI.

Levin, I. P., S. L. Schneider, and G. J. Gaeth (1998): All Frames Are Not Created Equal: A Typology and Critical Analysis of Framing Effects, Organizational Behavior and Human Decision Processes, 76(2), 149-188.

Levin, I. P., G. J. Gaeth, F. Evangelista, G. Albaum, and J. Schreiber (2001): How positive and negative frames influence the decisions of persons in the United States and Australia, Asia Pacific Journal of Marketing and Logistics,13(2), 64-71.

Liberman, V., S. Samuels, and L. Ross (2004): The Name of the Game: Predictive Power of Reputations vs. Situational Labels in Determining Prisoner's Dilemma Game Moves, Personality and Social Psychology Bulletin, 30, 1175-85.

McCusker, C., and P. J. Carnevale (1995): Framing in Resource Dilemmas: Loss Aversion and the Moderating Effects of Sanctions, Organizational Behavior and Human Decision Processes, 61, 190-201. Meyerowitz, B. E. and S. Chaiken (1987): The effect of message framing on breast self-examination attitudes, intentions, and behavior. Journal of Personality and Social Psychology, Vol. 52, 500-510.

Oosterbeek, H., R. Sloof, and G. van de Kuilen (2004): Cultural Differences in Ultimatum Game Experiments: Evidence from a Meta-Analysis, Experimental Economics, vol. 7(2), 171-188.

Park, E. S. (2000): Warm-glow versus cold-prickle: A further experimental study of framing effects on free-riding, Journal of Economic Behavior and Organization, 43, 405-421.

Pruitt, D. G. (1967): Reward structure and cooperation: the decomposed Prisoner's Dilemma game, Journal of Personality and Social Psychology, 7(1), 21-27.

Roth, A. E., V. Prasnikar, M. Okuno-Fujiwara, and S. Zamir (1991): Bargaining and market behavior in Jerusalem, Ljubljana, Pittsburgh, and Tokyo - An experimental study, American Economic Review, 81(5), 1068-1095.

Sell, J., Z.-Y. Chen, P. Hunter-Holmes, and A.C. Johansson (2002): A Cross-Cultural Comparison of Public Good and Resource Good Settings, Social Psychology Quarterly, 65(3), 285-297.

Selten, R. and C. C. Berg (1970): Drei experimentelle Oligopolserien mit kontinuierlichem Zeitverlauf, Beiträge zur experimentellen Wirtschaftsforschung, Vol. 2, 162-221.

Sonnemans, J., A. Schram, and T. Offerman (1998): Public good provision and public bad prevention: The effect of framing, Journal of Economic Behavior and Organization, Vol. 34, 143-161. 
Tversky, A. and D. Kahneman (1981): The framing of decisions and the psychology of choice, Science, Vol 211, Issue 4481, 453-458.

Willinger, M., and A. Ziegelmeyer (1999): Framing and cooperation in public goods games: An experiment with an interior solution, Economics letters, Vol. 65, 323-328. 


\section{Appendix}

\section{A External analogy with classical PD- and PG-games}

To show external analogy of both versions of our cooperation game with a classical binarychoice PD, we write down the $2 \times 2$-payoff matrix form of both designs including only the extreme points of total (e.g., $a_{i}^{P D P}=10 ; a_{i}^{P D N}=0$ ) and no cooperation (e.g., $a_{i}^{P D P}=$ $\left.0 ; a_{i}^{P D N}=10\right)$ :

Table 3

$2 \times 2$-matrix, representing the prisoner's dilemma game.

\begin{tabular}{|c|c|c|}
\hline$\pi_{\mathbf{1}}, \pi_{\mathbf{2}}$ & $\mathbf{C}_{\mathbf{2}}$ & $\mathbf{D}_{\mathbf{2}}$ \\
\hline $\mathbf{C}_{\mathbf{1}}$ & $k \cdot X, k \cdot X$ & $0, X+k \cdot X$ \\
\hline $\mathbf{D}_{\mathbf{1}}$ & $X+k \cdot X, 0$ & $X, X$ \\
\hline
\end{tabular}

The PD-condition $(1+k) \cdot X>k \cdot X>X>0$ is satisfied for all $k>1$ in both gameframes. In our experiment, this condition is fulfilled, with $k=2$. Given these parameters, by linear interpolation, payoffs from the discrete payoff matrix can be obtained. Having a freely pre-determined range of possible actions $a$ allows to obtain a non-binary measure of cooperation.

We now show external analogy of both game-frames with a typical PG-design. The payoff function of a common 2-person PG is given by:

$$
\pi_{i}^{P G}=X_{i}-a_{i}+k \cdot \frac{a_{i}+a_{j}}{2}, \text { with } i \neq j, \text { and } k>1
$$

$X_{i}$ represents player $i$ 's initial endowment. The parameter $a_{i}$ is the investment into the public good. Accordingly, $X_{i}-a_{i}$ represents the investment into the private good. All investments made to the public good are multiplied by the factor $k$. The fraction of one half of the increased public pie is returned to both players $i$ and $j$ by the addition to their investments into the private good. For $k<1$, it is rational for both players to invest nothing into the public good since the public pie shrinks. In the case of $k>1$, both players can increase their personal income by investing into the public good. However, in this case each player has a strong incentive to free-ride, hoping to reach even higher returns caused by a positive 
investment of the second player.

From the initial PG-equation, we get:

$$
\begin{gathered}
\pi_{i}=X_{i}-\left(1-\frac{k}{2}\right) \cdot a_{i}+k \cdot \frac{a_{j}}{2} \\
\Longleftrightarrow \pi_{i}=X_{i}-\theta \cdot a_{i}+k^{*} \cdot \theta \cdot a_{j}, \text { with } \theta=1-\frac{k}{2} \text {, and } k^{*}=\frac{k}{2 \cdot\left(1-\frac{k}{2}\right)}
\end{gathered}
$$

The payoff-function of the PDP-frame was given in equation by:

$$
\pi_{i}^{P D P}=X_{i}-a_{i}^{P D P}+k \cdot a_{j}^{P D P}
$$

It is evident that both game-frames are of the same type: a PG-game with parameter $k^{*}$ is formally similar to the PDP-frame with parameter $k$. Because of internal equivalence among $\mathrm{PDP}$ and PDN it is obvious that the PDN-frame is a PG, too. Contrary to the PG-game, in PDP and PDN there is no backflow of own investments. Thus, each $a_{i}>0$ is transferred directly to the opposite player, thereby providing a lower individual incentive to cooperate. 


\section{B Instructions for the experiment (for PDP and PDN)}

\section{B.1 Introduction}

Thank you for taking part in this experiment. Please read these instructions very carefully. It is very important that you do not talk to other participants for the time of the entire experiment. In case you do not understand some parts of the experiment, please read through these instructions again. If you have further questions after this, please give us a sign by raising your hand out of your cubicle. We will then approach you in order to answer your questions personally.

To guarantee your anonymity you will draw a personal code before the experiment starts. Please write this code on top of every sheet you use during this experiment. You will later receive your payment from this experiment by showing your personal code. This method ensures that we are not able to link your answers and decisions to you personally.

During this experiment you can earn money. The currency within the experiment is 'Taler'. The exchange rate from Taler to CURRENCY is:

\section{Taler $=$ XX CURRENCY}

Your personal income from the experiment depends on both your own decisions and on the decisions of other participants. Your personal income will be paid to you in cash as soon as the experiment is over.

During the course of the experiment, you will interact with a randomly assigned other participant. The assigned participant makes his/her decisions at the same point in time as you do. You will get no information on who this person actually is, neither during the experiment, nor at some point after the experiment. Similarly, the other participant will not be given any information about your identity. You will receive information about the assigned participant's decision after the entire experiment has ended.

After the experiment, please complete a short questionnaire, which we need for the statistical analysis of the experimental data. 


\section{B.2 Description of the experiment (PDP)}

In this experiment, you are randomly matched with another participant. You act as Person A, and the randomly assigned other participant acts as Person B. You and Person B must simultaneously make a similarly structured decision.

Person A and Person B first receive an initial endowment of 10 Talers.

You now have the opportunity to transfer any part of your endowment to Person B. You can only transfer integer amounts - thus, you can only choose amounts $a_{A} \in[\mathbf{0}, \mathbf{1}, \mathbf{2}, \mathbf{3}, \mathbf{4}, \mathbf{5}$, $6,7,8,9,10]$.

The amount you transfer to Person B is doubled. That means that Person B receives twice the amount you have transferred to him/her.

The randomly assigned participant acting as Person B is given exactly the same alternatives as you have. He/she also has the possibility to transfer any amount to you. The amount Person B transfers to you is also doubled. That means that you receive twice the amount Person B has transferred to you.

You will make your decisions simultaneously. During the course of the experiment, neither person receives any information concerning the decision of the other person.

\section{How the income is calculated}

Your personal income can be calculated as follows:

\begin{tabular}{|l|}
\hline Initial endowment \\
\hline - amount you choose to transfer to Person B \\
\hline + twice the amount $b$ Person B transferred to you \\
\hline \hline = your personal income \\
\hline
\end{tabular}




\section{B.3 Description of the experiment (PDN)}

In this experiment you are randomly matched with another participant. You act as Person A, and the randomly assigned other participant acts as Person B. You and Person B must simultaneously make a similarly structured decision.

Person A and Person B first receive an initial endowment of 10 Talers.

You now have the opportunity to transfer any part of Person B's endowment to yourself. You can only transfer integer amounts - thus, you can only choose amounts $a_{A} \in[\mathbf{0}, \mathbf{1}, \mathbf{2}, \mathbf{3}, \mathbf{4}$, $5,6,7,8,9,10]$.

The remaining amount - that is the amount that you do not transfer from Person B's endowment to yourself - is doubled. This means that Person B receives twice the amount that you do not transfer from him/her.

The randomly assigned participant acting as person B is given exactly the same alternatives as you have. He/she also has the possibility to transfer any amount to himself/herself. The remaining amount that he/she does not transfer from your endowment to himself/herself is doubled. This means that you receive twice the amount that he/she does not transfer from you.

You will make your decisions simultaneously. During the course of the experiment, neither person receives any information concerning the decision of the other person.

\section{How the income is calculated}

Your personal income can be calculated as follows:

\begin{tabular}{l}
\hline+ amount you choose to transfer from Person B to yourself \\
\hline+ twice the amount Person B did not transfer from your endowment \\
to himself/herself \\
\hline = your personal income
\end{tabular}

